\title{
Combination of Exercise and Intake of Amino Acid Mixture Synergistically Induces Beige Adipocyte Formation in Mice
}

\author{
Takuya KoJIMA, Nana ESAKI and Takanori TSUDA* \\ College of Bioscience and Biotechnology and Graduate School of Bioscience and Biotechnology, \\ Chubu University, Kasugai, Aichi 487-8501, Japan
}

(Received March 4, 2021)

\begin{abstract}
Summary Exercise combined with dietary factors may have significant effects on the suppression of body fat accumulation. Several trials suggest that amino acid mixtures containing alanine, arginine, and phenylalanine (ARF) combined with exercise can significantly reduce body fat accumulation in overweight adults and high-fat diet-induced obesity in mice. We therefore hypothesized that combining ARF and exercise would significantly induce beige adipocyte formation and that this would contribute to reducing body weight, whereas administration of ARF or exercise alone would not. Administration of ARF ( $1 \mathrm{~g} / \mathrm{kg}$ body weight, daily) combined with exercise ( 5 sessions per week) for 4 wk significantly induced formation of beige adipocytes in inguinal white adipose tissue (iWAT) in mice, although ARF or exercise alone did not. Metabolomic analysis showed that plasma lactate concentration was significantly elevated in the exercise+ARF group relative to the exercise group. Furthermore, lactate dehydrogenase B, which increases redox stress by converting lactate to pyruvate in iWAT and triggers induction of uncoupling protein 1 expression was significantly upregulated in iWAT of the exercise+ARF group. These findings demonstrate the unique effect of ARF combined with exercise for inducing beige adipocyte formation, which may be associated with the suggested lactate-mediated pathway. Appropriate mixtures of amino acids could be used as a dietary supplement before exercise and contributed to increasing energy expenditures.
\end{abstract}

Key Words alanine, arginine, brown-like adipocyte, phenylalanine, treadmill, metabolome, uncoupling protein 1

Exercise (EX) provides various benefits for overall metabolic health. Regular EX is a particularly effective strategy for body weight control because it elevates energy expenditure (1) and improves overall glucose and lipid homeostasis as well as insulin sensitivity (2, 3). EX combined with dietary factors may have significant or synergistic effects on increasing energy expenditure and suppression of body fat accumulation. For example, in a high-fat diet-induced obesity model in mice, a combination of EX and decaffeinated green tea

\footnotetext{
*To whom correspondence should be addressed.

E-mail: tsudat@isc.chubu.ac.jp

Abbreviations: ARF, amino acid mixture containing alanine, arginine, and phenylalanine; BAT, brown adipose tissue; CE-TOFMS, capillary electrophoresis time-of-flight mass spectrometry; EDL, extensor digitorum longus; eWAT, epididymal white adipose tissue; EX, exercise; FGF21, fibroblast growth factor 21; GAPDH, glyceraldehyde-3-phosphate dehydrogenase; H\&E, hematoxylin \& eosin; iWAT, inguinal white adipose tissue; LDHB, lactate dehydrogenase B; MCT1, monocarboxylic acid transporter 1; MCT4, monocarboxylic acid transporter 4; Nrf2, nuclear factor-erythroid 2-related factor 2; PGC-1 $\alpha$, peroxisome proliferator-activated receptor- $\gamma$ coactivator $1 \alpha$; scWAT, subcutaneous white adipose tissue; SNS, sympathetic nervous system; TA, tibialis anterior; TBP, TATA box binding protein; UCP1, uncoupling protein 1; WAT, white adipose tissue.
}

extract significantly decreased the accumulation of white adipose tissue (WAT) and improved insulin sensitivity (4). However, treatment with EX or decaffeinated green tea extract alone did not produce significant effects.

Another possible dietary factor that may offer improved health benefits when combined with EX is amino acids. There are many reports that dietary amino acid supplementation can modulate various metabolic changes $(5,6)$. Recent studies have shown that an amino acid mixture containing alanine, arginine, and phenylalanine (ARF) combined with EX significantly reduced body fat accumulation in overweight adults $(7$, 8 ) and diet-induced obesity in mice (9). However, there are few reports that other amino acid mixtures combined with EX reduced body fat accumulation, and the molecular mechanisms of the significant effect of ARF combined with EX on body fat in both obese mice and humans is not clear.

Mammals possess two types of adipose tissue, WAT and brown adipose tissue (BAT), which have physiologically distinct functions. WAT stores excess energy as triglycerides, whereas BAT releases excess energy through heat production from mitochondria (10). Thermogenesis in BAT requires the action of thermogenic uncoupling protein 1 (UCP1), which causes mitochondrial proton leak. Under these circumstances, heat is gener- 
ated instead of ATP (11). Brown-like adipocytes (also called beige adipocytes), which are induced in WAT, release excess energy as heat through a process mediated by UCP1, as in BAT (12). The induction of beige adipocyte can occur in response to $\beta 3$-adrenergic receptor agonists, full peroxisome proliferator-activated receptor $\gamma$ agonists, or simply chronic cold conditions $(11,13,14)$. Beige adipocyte induction is also a possible therapeutic target for treating obesity and various related disorders, and the development of beige adipocytes has also been linked by many studies to various dietary factors. In recent years, several published reports have shown that $\mathrm{EX}$ induces the formation of beige adipocytes in subcutaneous WAT (scWAT) in rodents $(15,16)$. In human studies, several reports have also indicated that high intensity-EX induces beige adipocyte formation in scWAT $(17,18)$. Based on these findings, it is likely that a combination of dietary factors and EX may effectively induce beige adipocyte formation in WAT, thus contributing to body weight control or providing other EX-mediated health benefits. However, little is known about how dietary factors interact with EX to induce beige adipocyte formation.

Accordingly, this study was conducted to examine whether ARF combined with EX significantly induces beige adipocyte formation and whether ARF administration or EX alone do not, as well as to clarify possible mechanisms by which ARF combined with EX mediate beige adipocyte formation.

\section{MATERIALS AND METHODS}

Chemicals. ARF was provided by Meiji Co., Ltd. (Tokyo, Japan). The composition of ARF was as follows (weight \%): L-alanine, 25\%; L-arginine, 25\%; L-phenylalanine; 50\%. Each amino acid (purity >99\%) was obtained from Kyowa Hakko Bio Co., Ltd. (Tokyo, Japan). Anti-UCP1 antibody (ab209483) was acquired from Abcam (Tokyo, Japan). Antibodies of monocarboxylate transporter 1 (MCT1, 20139-1A-AP), monocarboxylate transporter 4 (MCT4, 22787-1-AP), and lactate dehydrogenase B (LDHB, 19988-1-AP) were acquired from Proteintech (Tokyo, Japan). Anti- $\beta$-actin antibody (4967) was obtained from Cell Signaling Technology Japan (Tokyo, Japan). Anti-glyceraldehyde-3-phosphate dehydrogenase (GAPDH) antibody (016-25523) was acquired from FUJIFILM Wako Pure Chemical Corporation (Osaka, Japan).

Animal experiments. The design of the animal experiments was approved by the Animal Experiment Committee of Chubu University, and their guidelines were followed in the maintenance of all mice used in this work (Permission No. 3010024).

Effect of ARF combined with EX training on the formation of beige adipocytes in mice. Four-week-old male C57BL/6J mice were acquired from Japan SLC, Inc. (Hamamatsu, Japan), housed in an animal room at $23 \pm 3^{\circ} \mathrm{C}$ with a 12 -h light/dark cycle (illuminated 08:00-20:00), and provided free access to a standard laboratory diet (CE-2; CLEA Japan, Inc., Tokyo, Japan) and water (19-22). After $1 \mathrm{wk}$, mice were assigned to one of four groups ( $n=10$, for each group): sedentary control, sedentary control+ARF, EX, and EX+ARF. Mice received oral administration of either vehicle (saline, for control and EX groups) or ARF ( $1 \mathrm{~g} / \mathrm{kg}$ body weight, for ARF and $\mathrm{EX}+\mathrm{ARF}$ groups) daily for $4 \mathrm{wk}$. The dose of ARF was based on the results of a previous study (9) and a preliminary experiment to show that the administration level did not affect food intake. The $\mathrm{EX}$ and $\mathrm{EX}+\mathrm{ARF}$ group mice were trained for $60 \mathrm{~min}$, five times/wk for $4 \mathrm{wk}$ with a $0 \%$ incline treadmill (LE8710MTS; Panlab-Harvard Apparatus, Cornella, Spain, and KN-73; Natsume Seisakusho Co., Ltd., Tokyo, Japan). EX intensity for the five sessions per week was increased as follows: in the first week, $15 \mathrm{~m} / \mathrm{min}$; in the second week, $17.5 \mathrm{~m} / \mathrm{min}$; and in the final 2 week, $20 \mathrm{~m} / \mathrm{min}$. We performed a preliminary experiment to confirm that the EX intensity and duration did not induce beige adipocyte formation in inguinal WAT (iWAT) and UCP1 expression in BAT. During the experimental period, all mice groups were allowed free access to water and AIN-93G diet (23). At $16 \mathrm{~h}$ after the last EX training session and administration of saline or ARF, blood samples were collected from isoflurane-anesthetized mice, using a syringe containing heparin; plasma was subsequently isolated by centrifugation. Then, the iWAT, epididymal WAT (eWAT), interscapular brown adipose tissue (BAT), gastrocnemius + soleus muscle, and extensor digitorum longus (EDL) + tibialis anterior (TA) muscles were also removed (19-22). Following the protocol from our previous reports (19-22), small samples of adipose tissues were fixed and used for both UCP1 immunostaining and hematoxylin \& eosin (H\&E) staining. Aliquots of the tissues were also homogenized and used for immunoblotting analysis of UCP1 protein in iWAT and BAT, with GAPDH or $\beta$-actin used as controls, following the methods employed in our previous reports (19-22).

Metabolomic analysis. Metabolomic analyses were conducted on plasma from the $\mathrm{EX}$ and $\mathrm{EX}+\mathrm{ARF}$ groups using capillary electrophoresis time-of-flight mass spectrometry (CE-TOFMS) analysis with an Agilent CE-TOFMS system (Agilent Technologies, Tokyo, Japan) by Human Metabolome Technologies Inc. (Tsuruoka, Japan). Briefly, 50- $\mu \mathrm{L}$ plasma samples were extracted with $200 \mu \mathrm{L}$ of methanol and $20 \mu \mathrm{M}$ of internal standard (camphor-10-sulfonic acid, for anion analysis, and methionine sulfone, for cation analysis), to which $150 \mu \mathrm{L}$ of Milli-Q water was added before samples were mixed. The samples were filtered with a $5-\mathrm{kDa}$ cut-off filter at $9,100 \times g$ and $4^{\circ} \mathrm{C}$ for $120 \mathrm{~min}$. After filtrates were dried, they were dissolved in $50 \mu \mathrm{L}$ of water and subjected to CE-TOFMS analysis (24). Automatic integration software (MasterHands ver. 2.17.4.19, Keio University, Tsuruoka, Japan) was used to process the detected peaks, which were aligned based on their $\mathrm{m} / \mathrm{z}$ values, peak areas, and normalized migration times. Putative metabolites from the Human Metabolome Technologies metabolite database were used to annotate the peaks, internal standards were used to normalize peak areas, and comparisons of the peak areas against 
A
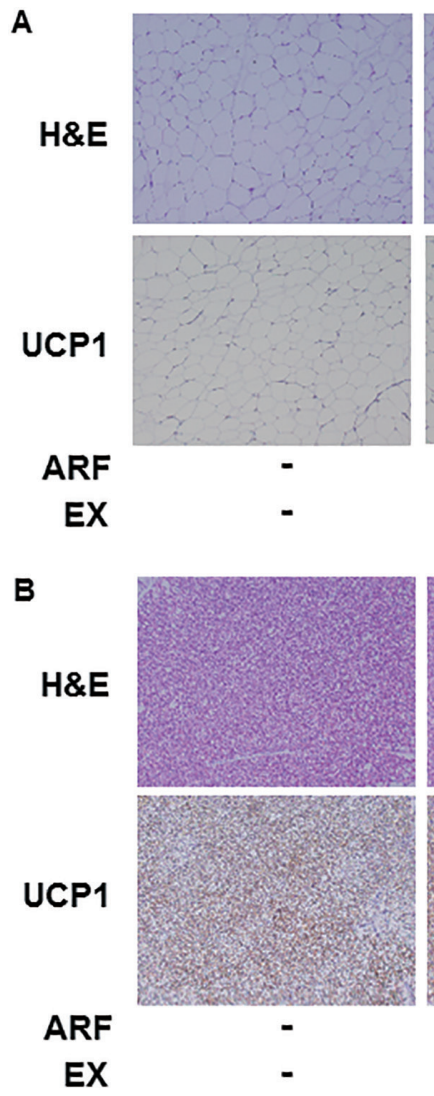
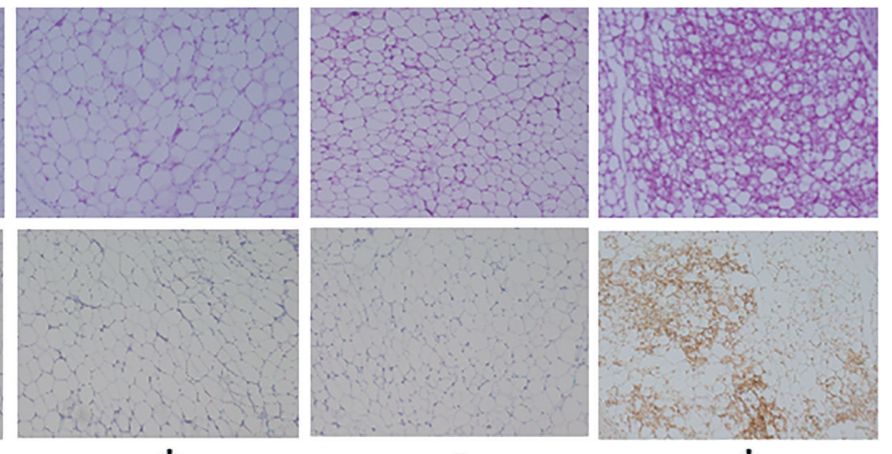

$+$

$-$
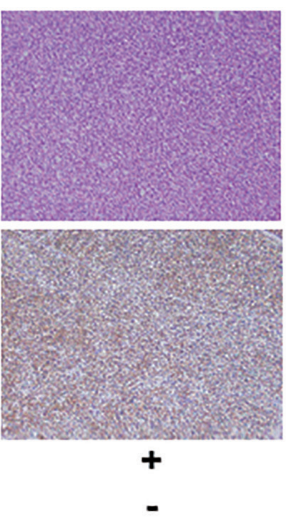
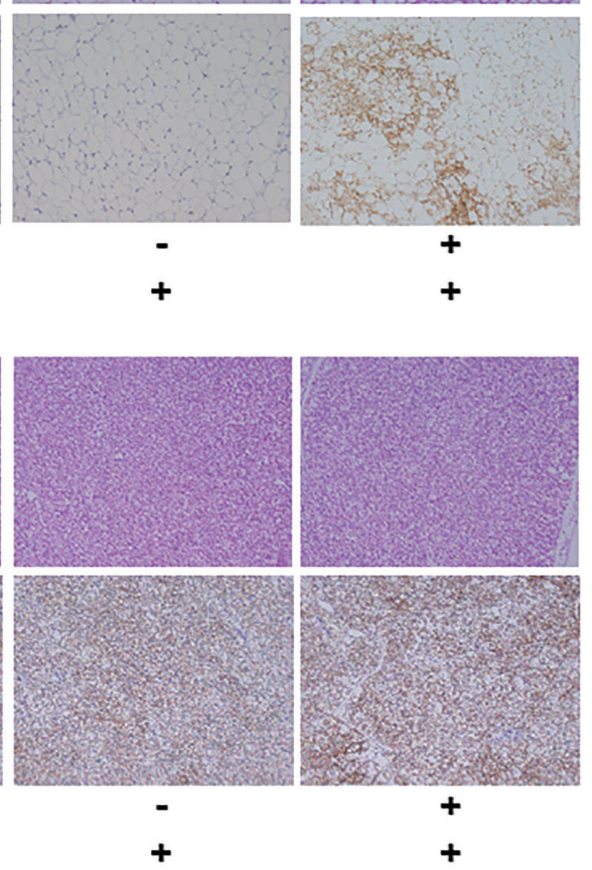

Fig. 1. Representative H\&E and immunohistochemical staining images of (A) iWAT and (B) BAT from mice in the control, ARF, EX, and EX+ARF groups for 4 wk.

calibration curves generated using internal standardization techniques were used to quantify the metabolites, thus enabling a comparison of the metabolites between the EX and EX+ARF groups.

Measurement of plasma fibroblast growth factor 21 (FGF21) concentration. Plasma FGF21 concentration was measured using an ELISA kit (Mouse/Rat FGF21 Quantikine ELISA Kit, R\&D Systems, Inc., Minneapolis, $\mathrm{MN}$ ) according to the manufacturer's instructions.

Measurement of mRNA levels. Total RNA isolation from the iWAT samples and mRNA transcript level assays with an ABI PRISM7300 real-time PCR system (Thermo Fisher Scientific, Yokohama, Japan) were performed following methods used by previous studies (19, 25). TaqMan Gene Expression Assays with the following ID numbers were used: MCT1 (gene name, Slc16a1), Mm01306379_m1; MCT4 (gene name, Slc16a3), Mm00446102_m1; LDHB, Mm00493146_m1; TATA box binding protein (TBP), Mm00446971_m1; FGF21, Mm00840165_g1.

Immunoblot analysis of MCT1, MCT4, and LDHB proteins. Immunoblot sample preparation from iWAT, gastrocnemius+soleus muscle, and EDL+TA muscle samples and immunoblot analysis was performed following methods used by previous studies (19-22, 25). Immunoreactivity for LDHB was visualized using Pierce Western Blotting Substrate (Thermo Fisher Scientific), and MCT1 and MCT4 immunoreactivity was visualized using ImmunoStar LD (FUJIFILM Wako Pure Chemical
Corporation) (19-22, 25).

Statistical analyses. All data are expressed as mean \pm SE values. The data presented in Fig. 2 and Table S1 (Supplemental Online Material) were analyzed using two-way ANOVA, followed by the Tukey-Kramer test with a $p<0.05$ significance threshold. Differences between two group means were assessed using Student's $t$-tests with a $p<0.05$ significance threshold (Table S2 (Supplemental Online Material), Figs. 3-5 and S2 (Supplemental Online Material)).

\section{RESULTS}

ARF combined with EX significantly induced beige adipocyte formation in iWAT

We first examined whether administration of ARF combined with EX for $4 \mathrm{wk}$ synergistically induced beige adipocyte formation compared with ARF or EX alone in mice. There were no significant interaction effects between the two treatments (ARF and EX) or differences among the four groups in body weight gain, food intake, adipose tissue (i.e., iWAT, eWAT, or BAT) or skeletal muscles weights (Table S1). Based on H\&Estained iWAT samples, the ARF alone and EX alone groups did not show clear visible multilocular adipocytes, indicating a lack of beige adipocytes, and UCP1 immunostaining was also negative (Fig. 1A). In contrast, distinct multilocular adipocytes were visible in H\&E stains of iWAT from the EX+ARF group. In addition, UCP1-immunopositive cells were also distinctly 
A

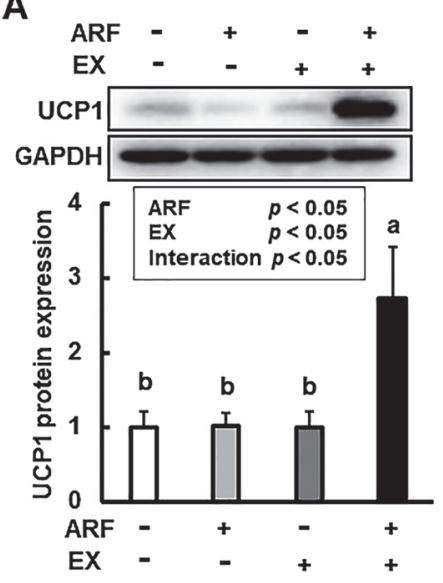

B

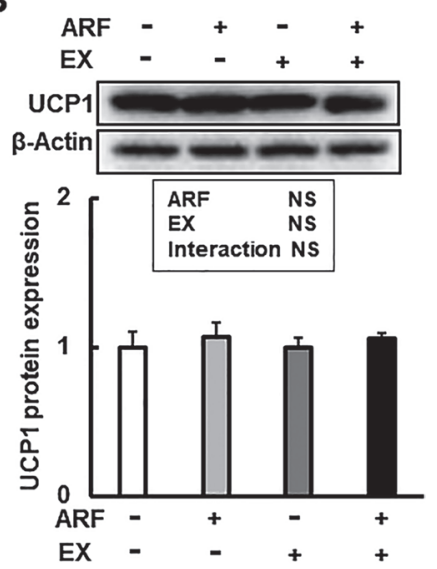

Fig. 2. UCP1 protein levels of (A) iWAT and (B) BAT from mice in the control, ARF, EX, and EX+ARF groups for 4 wk. The protein levels are expressed as fold-increases relative to the control group $(=1)$ after normalization with (A) GAPDH or (B) $\beta$-Actin expression levels. Data are presented as mean \pm SE values ( $n=9$ or 10$)$. The ARF $\times$ EX interaction effect in (A) was significant $(p<0.05)$; values in (A) that do not share a common letter differed significantly $(p<0.05)$. The $\mathrm{ARF} \times \mathrm{EX}$ interaction effect in (B) was not significant (NS).

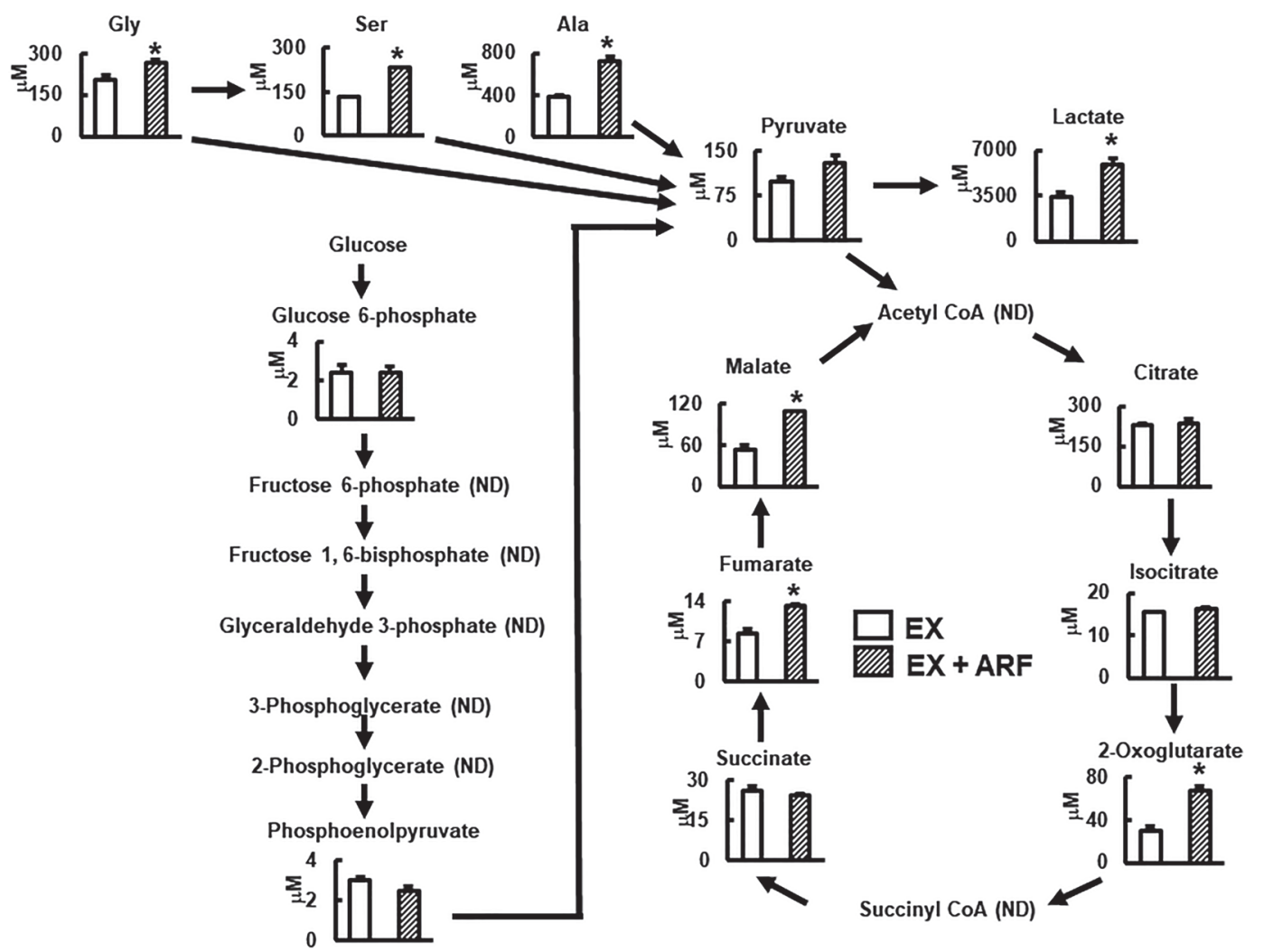

Fig. 3. The concentration of metabolites associated with glycolysis and the TCA cycle pathway in the plasma of mice in the EX and EX + ARF groups for $4 \mathrm{wk}$. Data are presented as mean \pm SE values $(n=3)$. * Significantly different compared with the EX group $(p<0.05)$; ND, not detected.

observed in iWAT from the EX and ARF group compared with the other groups (Fig. 1A). In eWAT from the four groups, there were no multilocular adipocytes, and UCP1 immunostaining was also negative (Fig. S1, Supplemental Online Material). In addition, the four groups did not differ in terms of H\&E staining or UCP1 immunostaining of BAT (Fig. 1B).

To confirm the significant immunohistochemical analysis results observed in the EX+ARF group, we examined the UCP1 protein levels in iWAT. Either administration of ARF alone or EX alone did not significantly induce UCP1 protein expression in iWAT (Fig. 2A). In contrast, the administration of ARF combined with EX significantly induced UCP1 protein expression in iWAT compared with the ARF and EX group (Fig. $2 \mathrm{~A})$. However, the UCP1 protein levels of BAT did not 
A

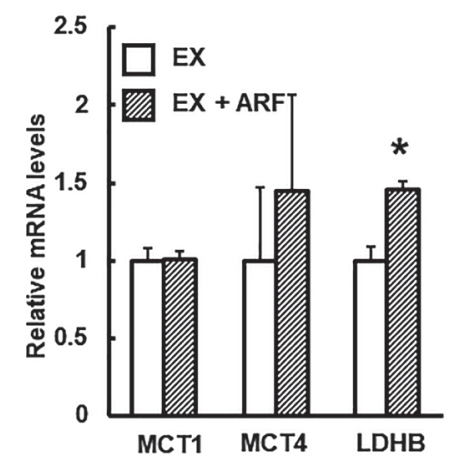

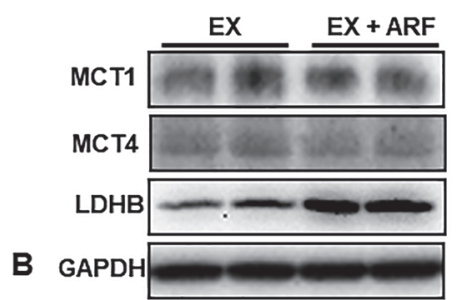

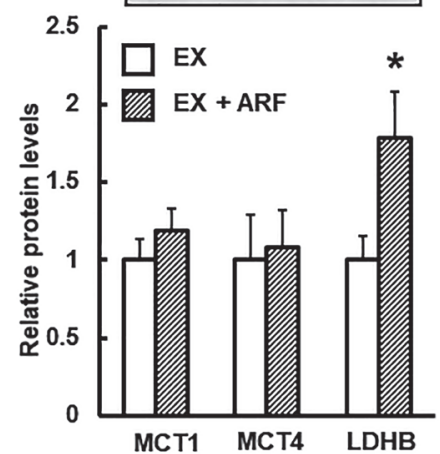

Fig. 4. The mRNA transcript (A) and protein levels (B) of MCT1, MCT4, and LDHB in iWAT of mice in the EX and EX+ARF groups for $4 \mathrm{wk}$. (A) MCT1, MCT4, and LDHB mRNA transcript levels are expressed as fold-increases relative to the EX group after normalization using TBP mRNA transcript levels. (B) MCT1, MCT4, and LDHB protein levels are expressed as fold-increases relative to the EX group $(=1)$ after normalization with GAPDH protein expression level. Data are presented as mean \pm SE values $(n=9)$. ${ }^{*}$ Significantly different compared with the EX group $(p<0.05)$.

A

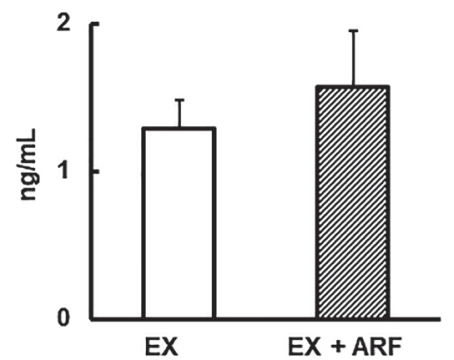

B

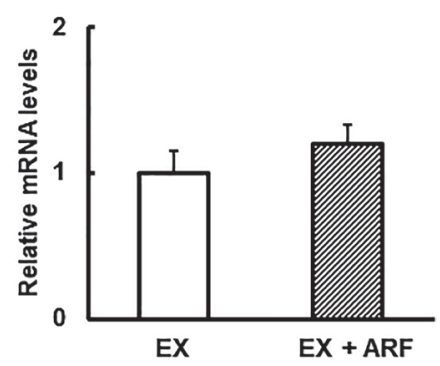

Fig. 5. The plasma concentrations (A) and mRNA transcript (B) of FGF21 in iWAT of mice in the EX and EX+ARF groups for $4 \mathrm{wk}$. (B) mRNA transcript levels are expressed as fold-increases relative to the EX group after normalization using TBP mRNA transcript levels. Data are presented as mean \pm SE values $(n=9)$.

differ among the four groups (Fig. 2B).

Plasma lactate and TCA cycle intermediates significantly increased under ARF combined with EX

The significant induction of beige adipocyte formation in iWAT of the EX+ARF group raised the issue of how ARF combines with EX training to induce beige adipocyte formation. To elucidate this mechanism, metabolic changes were assayed by CE-TOFMS in order to identify significantly differing levels of metabolites. Thus, we analyzed the metabolite profiles of plasma from the EX and EX+ARF groups and compared them. An overview of the plasma metabolite profiles revealed 186 peaks (118 cations and 68 anions) using the anion and cation modes of CE-TOFMS. Among the detected peaks, concentrations $(\mu \mathrm{M})$ of 58 peaks ( 42 cations and 16 anions) were calculated (Table S2). The metabolomic analysis clearly indicated significant changes had occurred in central carbon metabolism (i.e., glucose metabolism). As shown in Fig. 3, the concentrations of 2-oxoglutarate (2.2-fold), malate (2.1-fold), lactate (1.7-fold), and fumarate (1.6-fold) were significantly increased in the EX+ARF group compared with the EX group. Pyruvate was increased (1.3-fold) in the EX+ ARF group; however, the increase was not significant. In addition, the concentrations of Ala (1.9-fold), Ser (1.8-fold), and Gly (1.3-fold), which are converted into pyruvate and can further be metabolized into lactate, were significantly increased in the EX+ARF group.

ARF combined with EX significantly induced LDHB expression in iWAT

The metabolomic analysis indicated that plasma lactate was significantly increased in the EX+ARF group. Previous studies have demonstrated that lactate induces beige adipocytes mediated by a change in the intracellular redox state (26-28). Incorporated lactate via MCTs and/or intracellular lactate are metabolized into pyruvate by LDHB. The LDHB-mediated transformation induces a higher $\mathrm{NADH}: \mathrm{H}^{+} / \mathrm{NAD}^{+}$ratio and triggers 
upregulation of UCP1 expression to alleviate redox stress (26-28).

Accordingly, we examined the effect of ARF combined with EX on the expression of MCT1, MCT4, and LDHB in iWAT and muscles. The mRNA and protein expression levels of LDHB were significantly increased in the EX+ARF group compared with the EX group (Fig. 4A and B). The mRNA transcript and protein expression levels of MCT1 and MCT4 were not different between the groups (Fig. $4 \mathrm{~A}$ and B). In addition, the MCT1 and MCT4 protein expression levels were not different between the groups in either the gastrocnemius+ soleus muscle or EDL+TA muscle (Fig. S2A and B).

ARF combined with EX did not affect FGF21 plasma concentration or transcript abundance in iWAT

Previous studies have demonstrated that FGF21 induces the formation of beige adipocytes (29) and that EX significantly increases serum FGF21 concentration $(30,31)$. FGF21 is also expressed in WAT, and it is possible to induce beige adipocyte formation via autocrine or paracrine system (32). Therefore, we examined the effect of ARF combined with EX on the plasma FGF21 concentration and mRNA level in iWAT. The plasma FGF21 protein concentration and mRNA abundance in iWAT did not differ between the groups (Fig. 5A and B).

\section{DISCUSSION}

EX is considered an effective strategy for realizing various health benefits. However, it may be difficult for humans that have various forms of heart disease or physical disability to enhance their EX intensity. Accordingly, EX combined with dietary factors may be expected to increase energy expenditure and enhance other EX-related biological activity. Recent studies have demonstrated that ARF combined with EX can significantly reduce body fat accumulation in overweight adults $(7,8)$ and diet-induced obesity in mice (9). We hypothesized that a combination of ARF and EX would significantly induce beige adipocyte formation and may contribute to control of body weight.

After confirming that the EX intensity and duration did not affect beige adipocyte formation in iWAT, we examined whether a combination of ARF and EX significantly induce beige adipocyte formation. This study demonstrated that ARF administration combined with EX for 4 wk could significantly induce beige adipocyte formation in iWAT in mice, even though neither ARF administration nor EX alone had such an effect. The mice used in this study did not receive a high-fat diet nor do they represent a genetically induced obesity model. Therefore, it is not unexpected that body weight gain and WAT deposits did not differ among the groups. It is also reasonable that beige adipocyte formation is induced in iWAT but not in eWAT. PRD1-BF-1-RIZ1 homologous domain-containing protein-16, which is essential for the induction of beige adipocytes $(33,34)$, is highly expressed in scWAT (i.e., iWAT), but is expressed at an extremely low level in abdominal WAT (i.e., eWAT) (34). In addition, many studies have demonstrated that induction of beige adipocyte forma- tion in scWAT, not abdominal WAT, is significantly associated with a reduction of body fat accumulation and can be a potential therapeutic intervention against obesity (35-38) These previous findings and the current results suggest that the reduction of body fat accumulation promoted by ARF combined with EX in overweight adults $(7,8)$ and diet-induced obesity in mice (9) may be associated with the induction of beige adipocyte formation.

These results raise the issue of how ARF treatment combines with EX to induce the formation of beige adipocytes in iWAT of mice. One of the possible mechanisms, the $\beta 3$-adrenergic signaling pathway via the sympathetic nervous system (SNS), can induce UCP1 expression in iWAT and BAT (39). EX can stimulate SNS (40) and increase plasma norepinephrine concentrations (41). Furthermore, BAT is known to be more sensitive and dominant than WAT with respect to activation of the $\beta 3$-adrenergic signaling pathway via the SNS (42-44). If the $\beta 3$-adrenergic signaling pathway via the SNS is activated by ARF combined with EX, a significant induction of UCP1 expression should be observed in BAT from the ARF+EX group. However, in the present study, the UCP1 protein levels of BAT did not differ among the four groups. Therefore, it is unlikely that induction of beige adipocyte formation by ARF combined with EX is associated with activation of the $\beta 3$-adrenergic signaling pathway via the SNS.

Another possible factor underlying the mechanism of beige adipocyte induction by ARF combined with EX is FGF21. Previous studies have demonstrated that FGF21 induces the formation of beige adipocytes (29) and that EX significantly induces serum FGF21 concentration through upregulation of mRNA transcript abundance in the liver $(30,31)$. In addition, FGF21 is also expressed in WAT; however, FGF21 expressed in WAT acts locally via the autocrine or paracrine system rather than contributing to plasma concentrations of FGF21 (45-47). In the present study, plasma FGF21 concentrations did not differ between the EX and EX+ARF groups, suggesting that the $\mathrm{EX}+\mathrm{ARF}$ treatment does not affect FGF21 expression in the liver. In addition, FGF21 transcript abundances in iWAT did not differ between the EX and $\mathrm{EX}+\mathrm{ARF}$ groups. Although FGF21 may induce beige adipocyte formation in WAT via the autocrine or paracrine system (32), the present results suggest that induction of beige adipocyte formation ARF combined with EX is not associated with FGF21.

Metabolomic analysis is a particularly useful tool for exploring alternative factors of beige adipocyte induction by ARF combined with EX, and it can provide overviews of metabolic changes that may elucidate underlying physiological mechanisms. In the present study, no significant changes were observed in UCP1 expression and immunostaining between the ARF group compared with the control and EX groups. To determine how EX+ ARF induced beige adipocyte formation, we focused on the significant changes in plasma metabolites and several parameters in iWAT between the EX and EX+ARF groups. Although the metabolome results could be used 
to compare the two groups, focusing on the comparison between the two groups is sufficient to explain the possible mechanisms by which EX combined with ARF exerted a significant effect. Our metabolome results notably showed that the plasma concentration of lactate was significantly elevated in the $\mathrm{EX}+\mathrm{ARF}$ group compared with the EX group, despite this analysis having been conducted more than $16 \mathrm{~h}$ after the final EX session and ARF administration. Although we did not perform metabolomic analysis to compare the control and ARF groups, ARF alone did not significantly affect the plasma lactate concentration (control, 2.61土 $0.20 \mathrm{mM}$; ARF, 2.54 $\pm 0.21 \mathrm{mM}$ ). These results suggest that the plasma lactate concentration was significantly increased by the ARF combined with EX, not ARF alone. Lactate has been long considered a waste product of anaerobic metabolism. However, lactate production is now regarded to enhance glucose metabolism under aerobic conditions $(48,49)$, suggesting that ARF combined with EX accelerates glucose metabolism. Carrière et al. demonstrated that lactate induces beige adipocyte formation mediated through a change in the intracellular redox state (26-28). In adipocytes, lactate is metabolized into pyruvate by $\mathrm{LDHB}$ (lactate $+\mathrm{NAD}^{+} \rightarrow$ pyruvate $+\mathrm{NADH}+\mathrm{H}^{+}$); this $\mathrm{LDHB}-$ mediated transformation induces a higher $\mathrm{NADH}: \mathrm{H}^{+} / \mathrm{NAD}^{+}$ratio (i.e., a high redox state) (26-28). This increase triggers induction of UCP1 expression via redox-sensitive signaling pathways (50-52). The $\mathrm{NADH}: \mathrm{H}^{+} / \mathrm{NAD}^{+}$ratio triggered elevation of mitochondrial redox state may be associated with nuclear factor-erythroid 2-related factor 2 (Nrf2), a transcription factor controlling a broad range of reactive oxygen species and thiol targeted antioxidant enzymes $(52,53)$. Significant induction of UCP1 expression was observed in iWAT, but administration of $\mathrm{N}$-acetylcysteine suppressed the induction in Nrf2 knock out mice (53). As a result, uncoupling activity mediated by $\mathrm{UCP} 1$ promotes electron transport chain activity, resulting in a decrease in the $\mathrm{NADH}: \mathrm{H}^{+} / \mathrm{NAD}^{+}$ratio, which alleviates redox stress (26-28).

In this study, the plasma lactate concentration was significantly elevated in the EX+ARF group compared with the EX group. Furthermore, the LDHB protein expression level was significantly increased in iWAT from the EX+ARF group compared with that from the EX group. This significant increase may be associated with the beige adipocyte formation induced by the combination of ARF and EX, that is, lactate can be converted into pyruvate by LDHB and facilitates elevation of mitochondrial redox state through increase in $\mathrm{NADH}: \mathrm{H}^{+} / \mathrm{NAD}^{+}$ratio, thus $\mathrm{UCP} 1$ is induced in iWAT from the EX+ARF group. Nrf2 can be associated with the induction in the EX+ARF group (53). Peroxisome proliferator-activated receptor- $\gamma$ coactivator $1 \alpha$ (PGC$1 \alpha$ ) is one of the key factors of metabolic controls. Summermatter et al. reported that PGC- $1 \alpha$ promotes LDHB transcription by coactivating estrogen-related receptor- $\alpha$ on LDHB promoter (54). ARF administration combined with EX may modulate PGC- $1 \alpha$ activity and result in upregulation of LDHB in iWAT. In addition, concen- trations of Ala, Gly, and Ser, which can be converted into pyruvate and form lactate, were significantly increased in the EX+ARF group compared with the EX group. These significant increases may have contributed to the significant elevation of the plasma lactate concentration observed in the EX+ARF group.

Our metabolome results also showed that the plasma concentrations of Arg, Phe, 2-oxoglutarate, and fumarate were significantly elevated in the $\mathrm{EX}+\mathrm{ARF}$ group compared with the EX group. Increases in Arg and Phe content could be converted into 2-oxoglutarate or fumarate, and this conversion may be associated with significant increases in plasma 2-oxoglutarate and fumarate concentrations in the $\mathrm{EX}+\mathrm{ARF}$ group.

The results of this study raise some unaddressed questions and key limitations. One report showed that 2-oxoglutarate induced beige adipocyte formation in WAT in mice (55). Although 2-oxoglutarate may be another possible factor affecting beige adipocyte induction by ARF combined with EX, we did not investigate whether the increased plasma 2-oxoglutarate content mediated beige adipocyte formation in the $\mathrm{EX}+\mathrm{ARF}$ group. In addition, because only small amounts of iWAT were obtained from the mice, we were unable to determine $\mathrm{NADH}: \mathrm{H}^{+} / \mathrm{NAD}^{+}$ratio and lactate content in iWAT. In future research, using iWAT-specific LDHB knockout mice or inducing lactate depletion with chemical inhibitors may be useful tools for confirming lactate-mediated beige adipocyte formation is induced by ARF combined with EX.

In conclusion, we have demonstrated that ARF administration combined with EX for 4 wk can significantly induce beige adipocyte formation in iWAT of mice, even though ARF or EX alone did not have this effect. A lactate-mediated pathway of beige adipocyte formation may be driven by administration of ARF combined with EX. These findings demonstrate that the combination of ARF with EX offers a unique biological response for inducing beige adipocyte formation, which may be involved with increasing energy expenditures.

\section{Authorship}

TT and TK designed this research; TK and NE performed the experiments; TT wrote the paper.

\section{Disclosure of state of COI \\ No conflicts of interest to be declared.}

\section{Acknowledgments}

We thank Naomi Tagami and Sho Nishikawa (Chubu University) for their technical assistance and helpful discussion.

This study was supported in part by Grants-in-Aid for Scientific Research (Nos. 18H02157, 18KK0165, 20K05946, 20H02942 to Takanori Tsuda) from the Japan Society for Promotion of Science and the Food Science Institute Foundation (Ryoushoku-Kenkyukai to Takanori Tsuda). 
Supporting information

Supplemental online material is available on J-STAGE.

\section{REFERENCES}

1) Jakicic JM. 2003. Exercise in the treatment of obesity. Endocrinol Metab Clin North Am 32: 967-980.

2) Joyner MJ, Green DJ. 2009. Exercise protects the cardiovascular system: effects beyond traditional risk factors. $J$ Physiol 587: 5551-5558.

3) Goodyear LJ, Kahn BB. 1998. Exercise, glucose transport, and insulin sensitivity. Ann Rev Med 49: 235-261.

4) Tan SS, Rogers CJ, Lambert JD. 2014. Voluntary exercise and green tea enhance the expression of genes related to energy utilization and attenuate metabolic syndrome in high fat fed mice. Mol Nutr Food Res 58: 1156-1159.

5) Gannon MC, Nuttall JA, Nuttall FQ. 2002. The metabolic response to ingested glycine. Am J Clin Nutr 76: 1302-1307.

6) Commisotto PG, Gélinas Y, Deshaies Y, Bukowiecki LJ. 2005. Regulation of leptin secretion from white adipocytes by insulin, glycolytic substrates, and amino acids. Am J Physiol Endcrinol Metab 289: E166-E171.

7) Sasai H, Ueda K, Tsujimoto T, Kobayashi H, Sanbongi C, Ikegami S, Nakata Y. 2017. Dose-ranging pilot randomized trial of amino acid mixture combined with physical activity promotion for reducing abdominal fat in overweight adults. Diabetes Metab Syndr Obes 10: 297-309.

8) Ueda K, Sasai H, Tsujimoto T, Sanbongi C, Ikegami S, Kobayashi H, Shioya N, Suzuki S, Nakata Y. 2018. Randomized trial of amino acid mixture combined with physical activity promotion for abdominal fat reduction in overweight adults. Diabetes Metab Syndr Obes 11: 23-33.

9) Ueda K, Sanbongi C, Takai S, Ikegami S. 2017. A combination of exercise and amino acid mixture supplementation comprised of arginine, alanine and phenylalanine reduces diet-induced obesity in mice. Jpn Pharmacol Ther 45: 1289-1294.

10) Giralt M, Villarroya F. 2013. White, brown, beige/brite: different adipose cells for different functions? Endocrinology 154: 2992-3000.

11) Cannon B, Nedergaard J. 2004. Brown adipose tissue: function and physiological significance. Physiol Rev 84: 277-359.

12) Ishibashi J, Seale P. 2010. Beige can be slimming. Science 328: 1113-1114.

13) Nagase I, Yoshida T, Kumamoto K, Umekawa T, Sakane N, Nikami H, Kawada T, Saito M. 1996. Expression of uncoupling protein in skeletal muscle and white fat of obese mice treated with thermogenic beta 3-adrenergic agonist. J Clin Invest 97: 2898-2904.

14) Petrovic N, Walden TB, Shabalina IG, Timmons JA, Cannon B, Nedergaard J. 2010. Chronic peroxisome proliferator-activated receptor gamma (PPARgamma) activation of epididymally derived white adipocyte cultures reveals a population of thermogenically competent, UCP1-containing adipocytes molecularly distinct from classic brown adipocytes. J Biol Chem 285: 7153-7164.

15) Stanford K, Middelbeek IRJ, Townsend KL, Lee MY, Takahashi H, So K, Hitchcox KM, Markan KR, Hellbach K, Hirshman MF, Tseng YH, Goodyear LJ. 2015. A novel role for subcutaneous adipose tissue in exercise-induced improvements in glucose homeostasis. Diabetes 64: 2002-2014.

16) Cao L, Choi EY, Liu X, Martin A, Wang C, Xu X, During
MJ. 2011. White to brown fat phenotypic switch induced by genetic and environmental activation of a hypothalamic-adipocyte axis. Cell Metab 14: 324-338.

17) Norheim F, Langleite TM, Hjorth M, Holen T, Kielland A, Stadheim HK, Gulseth HL, Birkeland KI, Jensen J, Drevon CA. 2014. The effects of acute and chronic exercise on PGC- $1 \alpha$, irisin and browning of subcutaneous adipose tissue in humans. FEBS J 281: 739-749.

18) Díaz BO, Flores MR, Muñoz VS, Preciado FM, Ortega SO, Elias VB, Guzmán GB, Monge RO, García EG, González BP, Molina MTV, Salazar MS, Puente BA. 2018. Exercise induces white adipose tissue browning across the weight spectrum in humans. Front Physiol 9: 1781.

19) Nishikawa S, Aoyama H, Kamiya M, Higuchi J, Kato A, Soga M, Kawai T, Yoshimura K, Kumazawa S, Tsuda T. 2016. Artepillin C, a typical Brazilian propolis-derived component, induces brown-like adipocyte formation in C3H10T1/2 cells, primary inguinal white adipose tissue-derived adipocytes, and mice. PLoS One 11: e0162512.

20) Nishikawa S, Hyodo T, Nagao T, Nakanishi A, Tandia M, Tsuda T. 2019. $\alpha$-Monoglucosyl hesperidin but not hesperidin induces brown-like adipocyte formation and suppresses white adipose tissue accumulation in mice. $J$ Agric Food Chem 67: 1948-1954.

21) Nishikawa S, Kamiya M, Aoyama H, Nomura M, Hyodo T, Ozeki A, Lee H, Takahashi T, Imaizumi A, Tsuda T. 2018. Highly dispersible and bioavailable curcumin but not native curcumin induces brown-like adipocyte formation in mice. Mol Nutr Food Res 62: 1700731.

22) Nishikawa S, Hyodo T, Aoyama H, Miyata R, Kumazawa S, Tsuda T. 2020. Artepillin C, a key component of Brazilian propolis, induces thermogenesis in inguinal white adipose tissue of mice through a creatine-metabolism-related thermogenic pathway. J Agric Food Chem 68: 1007-1014.

23) Reeves PG, Nielsen FH, Fahey Jr GC. 1993. AIN-93 purified diets for laboratory rodents: final report of the American Institute of Nutrition ad hoc writing committee on the reformulation of the AIN-76A rodent diet. $J$ Nutr 123: 1939-1951.

24) Uchiwa T, Takai Y, Tashiro A, Furuse M, Yasuo S. 2016. Exposure of C57BL/6J mice to long photoperiod during early life stages increases body weight and alters plasma metabolomic profiles in adulthood. Physiol Rep 4: e12974.

25) Takikawa M, Inoue S, Horio F, Tsuda T. 2010. Dietary anthocyanin-rich bilberry extract ameliorates hyperglycemia and insulin sensitivity via activation of AMP-activated protein kinase in diabetic mice. J Nutr 140: 527533.

26) Carrière A, Jeanson Y, Berger-Muller S, André M, Chenouard V, Arnaud E, Barreau C, Walther R, Galinier A, Wdziekonski B, Villageois P, Louche K, Collas P, Moro C, Dani C, Villarroya F, Casteilla L. 2014. Browning of white adipose cells by intermediate metabolites: an adaptive mechanism to alleviate redox pressure. Diabetes 63: 3253-3265.

27) Jeanson Y, Carrière A, Casteilla L. 2015. A new role for browning as a redox and stress adaptive mechanism? Front Endocrinol 6: 158.

28) Carrière A, Lagarde D, Jeanson Y, Portais JC, Galinier A, Ader I, Casteilla L. 2020. The emerging roles of lactate as a redox substrate and signaling molecule in adipose tissues. J Physiol Biochem 76: 241-250. 
29) Fisher FM, Kleiner S, Douris N, Fox EC, Mepani RJ, Verdeguer F, Wu J, Kharitonenkov A, Flier JS, Flier EM, Spiegelman BM. 2012. FGF21 regulates PGC- $1 \alpha$ and browning of white adipose tissues in adaptive thermogenesis. Genes Dev 26: 271-281.

30) Tanimura Y, Aoi W, Takanami Y, Kawai Y, Mizushima K, Naito Y, Yoshikawa T. 2016. Acute exercise increases fibroblast growth factor 21 in metabolic organs and circulation. Physiol Rep 4: e12828.

31) Kim KH, Kim SH, Min YK, Yang HM, Lee JB, Lee MS. 2013. Acute exercise induces FGF21 expression in mice and in healthy humans. PLoS One 8: e63517.

32) López TQ, Cereijo R, Turatsinze JV, Planavila A, Cairó M, Navarro AG, Peyrou M, Moure R, Iglesias R, Giralt M, Eizirik DL, Villarroya F. 2016. The lipid sensor GPR120 promotes brown fat activation and FGF21 release from adipocytes. Nat Commun 7: 13479.

33) Seale P, Conroe HM, Estall J, Kajimura S, Frontini A, Ishibashi J, Cohen P, Cinti S, Spiegelman BM. 2011. Prdm16 determines the thermogenic program of subcutaneous white adipose tissue in mice. J Clin Invest 121: 96-105.

34) Cohen P, Levy JD, Zhang Y, Frontini A, Kolodin DP, Svensson KJ, Lo JC, Zeng X, Ye L, Khandekar MJ, Wu J, Gunawardana SC, Banks AS, Camporez JPG, Jurczak MJ, Kajimura S, Piston DW, Mathis D, Cinti S, Shulman GI, Seale P, Spiegelman BM. 2014. Ablation of PRDM16 and beige adipose causes metabolic dysfunction and a subcutaneous to visceral fat switch. Cell 156: 304-316.

35) Saito M, Okamatsu-Ogura Y, Matsushita M, Watanabe K, Yoneshiro T, Nio-Kobayashi J, Iwanaga T, Miyagawa M, Kameya T, Nakada K, Kawai Y, Tsujisaki M. 2009. High incidence of metabolically active brown adipose tissue in healthy adult humans. Diabetes 58: 15261531.

36) van Marken Lichtenbelt WD, Vanhommerig JW, Smulders NM, Drossaerts JM, Kemerink GJ, Bouvy ND, Schrauwen P, Teule GJ. 2009. Cold-activated brown adipose tissue in healthy men. $N$ Enlg J Med 360: 15001508.

37) Nedergaard J, Cannon B. 2010. The changed metabolic world with human brown adipose tissue: therapeutic visions. Cell Metab 11: 268-272.

38) Yoneshiro T, Aita S, Matsushita M, Kameya T, Nakada K, Kawai Y, Saito M. 2011. Brown adipose tissue, wholebody energy expenditure, and thermogenesis in healthy adult men. Obesity 19: 13-16.

39) Yang X, Ruan H. 2015. Neuronal control of adaptive thermogenesis. Front Endocrinol 6: 1-9.

40) Dimsdale JE, Moss J. 1980. Plasma catecholamines in stress and exercise. JAMA 243: 340-342.

41) Christensen NJ, Galbo H, Hansen JF, Hesse B, Richter EA, Trap-Jensen J. 1979. Catecholamines and exercise. Diabetes 28 (Suppl 1): 58-62.

42) Slavin BG, Ballard KW. 1978. Morphological studies on the adrenergic innervation of white adipose tissue. Anat Rec 191: 377-389.
43) Trayhurn P, Ashwell M. 1987. Control of white and brown adipose tissues by the autonomic nervous system. Proc Nutr Soc 46: 135-142.

44) Zouhal H, Jacob C, Delamarche P, Gratas-Delamarche A. 2008. Catecholamines and the effects of exercise, training and gender. Sports Med 38: 401-423.

45) Dutchak PA, Katafuchi T, Bookout AL, Choi JH, Yu RT, Mangelsdorf DJ, Kliewer SA. 2012. Fibroblast growth factor-21 regulates PPARgamma activity and the antidiabetic actions of thiazolidinediones. Cell 148: 556567.

46) BonDurant LD, Potthoff MJ. 2018. Fibroblast growth factor 21: a versatile regulator of metabolic homeostasis. Annu Rev Nutr 38: 173-196.

47) van Baak MA, Vink RG, Roumans NJT, Cheng CC, Adams AC, Mariman ECM. 2020. Adipose tissue contribution to plasma fibroblast growth factor 21 and fibroblast activation protein in obesity. Int J Obes 44: 544 547.

48) Katz A, Sahlin K. 1988. Regulation of lactic acid production during exercise. J Appl Physiol 65: 509-518.

49) Kemper WF, Lindstedt SL, Hartzler LK, Hicks JW, Conley KE. 2001. Shaking up glycolysis: Sustained, high lactate flux during aerobic rattling. Proc Natl Acad Sci USA 98: 723-728.

50) Chouchani ET, Kazak L, Jedrychowski MP, Lu GZ, Erickson BK, Szpyt J, Pierce KA, Laznik-Bogoslavski D, Vetrivelan R, Clish CB, Robinson AJ, Gygi SP, Spiegelman BM. 2016. Mitochondrial ROS regulate thermogenic energy expenditure and sulfenylation of UCP1. Nature 532: 112-116.

51) Han YH, Buffolo M, Pires KM, Pei S, Scherer PE, Boudina S. 2016. Adipocyte-specific deletion of manganese superoxide dismutase protects from diet-induced obesity through increased mitochondrial uncoupling and biogenesis. Diabetes 65: 2639-2651.

52) Chouchani ET, Kazak L, Spiegelman BM. 2017. Mitochondrial reactive oxygen species and adipose tissue thermogenesis: Bridging physiology and mechanisms. J Biol Chem 292: 16810-16816.

53) Schneider K, Valdez J, Nguyen J, Vawter M, Galke B, Kurtz TW, Chan JY. 2016. Increased energy expenditure, Ucp1 expression, and resistance to diet-induced obesity in mice lacking nuclear factor-erythroid-2-related transcription factor-2 (Nrf2). J Biol Chem 291: 7754-7766.

54) Summermatter S, Santos G, Pérez-Schindler J, Handschin C. 2013. Skeletal muscle PGC- $1 \alpha$ controls wholebody lactate homeostasis through estrogen-related receptor $\alpha$-dependent activation of $\mathrm{LDH} \mathrm{B}$ and repression of LDH A. Proc Natl Acad Sci USA 110: 87388743.

55) Tian Q, Zhao J, Yang Q, Wang B, Deavila JM, Zhu MJ, Du M. 2020. Dietary alpha-ketoglutarate promotes beige adipogenesis and prevents obesity in middle-aged mice. Aging Cell 19: e13059. 